\title{
Reducing the risks of drug use: The case for set and setting
}

\author{
PHIL DALGARNO ${ }^{1}$, \& DAVID SHEWAN ${ }^{2}$ \\ ${ }^{1}$ Nursing, Midwifery and Allied Health Professions Research Unit, Glasgow Caledonian University, \\ City Campus, Cowcaddens Road, Glasgow, G4 OBA and ${ }^{2}$ Glasgow Centre for the Study of Violence, \\ Department of Psychology, Glasgow Caledonian University, City Campus, Cowcaddens Road, \\ Glasgow, G4 OBA
}

(Received 2002; accepted 6 December 2004)

\begin{abstract}
The central proposition of this article is that if people are thoughtful, well-prepared and aware of the means and best environments for using a particular drug, then the risks associated with the use of a particular drug - any drug - can be minimal. The types of drugs discussed in this context focus on those assumed to be the most 'addictive' - heroin and cocaine - to those less well-known but potentially more hazardous to use without prior knowledge and preparation - such as Yage and Fly Agaric. This proposition is discussed in the context of different definitions of relatively non-problematic patterns of drug use, specifically: controlled, recreational and unobtrusive. It is concluded that while the effect of taking a particular drug is a primary motivation to the user, the role of set and setting are of fundamental importance in ensuring that the effects of that drug are as intended for and expected by the user.
\end{abstract}

Keywords: Controlled drug use, set and setting, addictive, risk

\section{Introduction}

The central proposition of this article is that drug use need not be dangerous. Put simply, if people are thoughtful, well-prepared, and aware of the means to minimize the risks associated with the use of a particular drug, then using that drug - any drug - need not be dangerous. ${ }^{1}$ Indeed the self-reported outcomes from drug use of this type can range from enjoyment, to relaxation, to problem solving and self-exploration. This will be regarded as a bold statement. Therefore, we will seek to justify it with reference to theories of drug use, and in the context of empirical evidence for controlled, unobtrusive, recreational use of drugs that can be regarded as having the most potential for dangerousness.

Correspondence: Phil Dalgarno, Nursing, Midwifery and Allied Health Professions Research Unit, Glasgow Caledonian University, City Campus, Cowcaddens Road, Glasgow, G4 0BA, UK. Tel: 0141 331 8105. Fax: 01413318101. E-mail: pda@gcal.ac.uk

${ }^{1}$ This is not in any sense to imply that drug use is safe: there are very obvious risks associated with underlying physical and/or mental health problems, and of course one can never rule out allergic reactions. 
What we mean when we talk about psychoactive drugs is apparently straightforward. It is customary to describe the effects of specific psychoactive substances with the assumption that these drugs affect different people in the same way. The chemical and neurological effects of psychoactive drugs can be defined and explained (Wise, 1990), but confusion and controversy can arise when these effects are equated with a capacity intrinsic to drugs to affect human experience and behaviour in a consistent and entirely predictable way (Alexander, 2000; Davies, 1992; Falk, 1983; Hammersley \& Reid, 2001; Schaler, 2000). Such an assumption has been described as "...widespread, but it remains a fallacy" (Gossop, 1993, p. 15). Similarly, a set of assumptions exists as to the long-term effects of drugs, with certain drugs believed to have the capacity to lead to inevitably destructive patterns of use. Several studies have provided evidence for controlled cocaine use, where cocaine, a drug occupying a position close to the top of the hierarchy of dangerous substances (National Institute on Drug Abuse, 2000) is used in a largely non-problematic way (Bielman, Diaz, Merlo \& Kaplan, 1993; Cohen \& Sas, 1994; Hammersley \& Ditton, 1994; Mugford, 1994; Reinarman, Murphy \& Waldorf, 1994; Waldorf, Reinarman \& Murphy, 1991). Though less researched, evidence is emerging that heroin, regarded as one of the most addictive and destructive drugs, can also be used in a way that is not associated with either serious health problems nor high levels of criminal behaviour, with much of this argument deriving from Zinberg's original work (Zinberg, 1984), with support from Blackwell's rather neglected paper (Blackwell, 1983), and more recent empirical work (Shewan \& Dalgarno, 2005; Shewan et al., 1998), and encompassing a growing body of theoretical literature (Alexander, 2000; Robins, Compton \& Horton, 2000; Schaler, 2000). The rather dull theme running through empirical literature in this area is the simple testing and confirmation of the null hypothesis; Question: "Is cocaine/heroin an intrinsically dangerous drug?" Answer: "Not necessarily". In principle, therefore, it should also be possible to use crack cocaine in a relatively non-problematic way. The argument that it should be possible to use all psychoactive drugs in a 'controlled' fashion provides the theoretical basis for this article, according equal importance to psychological and social factors, as well as pharmacological effects, as influences on patterns of drug use. ${ }^{2}$

Of course, delving into drug theory very quickly raises issues regarding what Alexander (1994) describes as 'the labyrinth' of drug terminology. Controlled is the term most commonly used to describe drug use that troubles neither health services nor criminal justice agencies. While we have no huge opposition to this term, it does raise a number of issues concerning definition. Specifically, "controlled by whom?" is one example of the ambiguity around the term. Is a strict and well-adhered to prescribing regime, of methadone for example, similar in terms of control to the studies on cocaine and heroin use described above? Clearly not, most would argue. Yet both models rely on a degree of self-control on behalf of the user. Within the prescribing regime, however, it could be argued that the 'real' control lies in the hands of the doctor signing the prescriptions, whereas a nonproblematic cocaine and heroin user is often at the mercy of the vagaries of the illegal drug market, in terms of availability, purity and not to mention being caught engaging in an illegal activity with the concomitant risks of a criminal record and possibly even imprisonment. So, controlled drug use as a definition would appear to confirm Alexander's position.

An alternative phrase for non-problematic drug use is that of recreational use. However, since its introduction to the labyrinth of drug terminology (Goode, 1969), it has brought

\footnotetext{
${ }^{2}$ In other words, drug, set and setting.
} 
with it a potential for misunderstanding and misinterpretation. We have our reservations about this term. Firstly, the term is predominantly used for a particular 'group' of drugs, such as cannabis, ecstasy and LSD, these being drugs not generally associated with destructive or addictive use, due to the relatively low level of medical intervention and criminal behaviour associated with such drugs. A questioning of the pharmacological model of addiction and recognition of the importance of psychological factors leads to an acceptance that, in theory, problematic and non-problematic use can occur with the whole range of psychoactive substances (Davies, 1992; Reinarman et al., 1994; Shewan et al., 1998; Zinberg, 1984). Thus, while controlled use of heroin, and indeed crack cocaine, should be demonstrable, so should addicted patterns of use of the 'softer' drugs (Hammersley \& Reid, 2002). As such, according the principle of recreational use only to recreational drugs is misleading.

If applied only to certain drugs, recreational drug use reinforces the concept of there being an addictive hierarchy of substances. Therefore ecstasy is 'fun' whereas heroin is not: ecstasy is used on an occasional, leisure time basis, whereas heroin is used with monotonous regularity through necessity. The research on controlled heroin use would dispute this view, and indeed in the authors' own research on heroin use participants typically described their motivation for using heroin in hedonistic terms: they enjoyed the effects (Shewan \& Dalgarno, 2005; Shewan et al., 1998). Yet 'recreational heroin use' has been viewed as a subversive, even irresponsible term: “...The opiates are drugs of addiction. There are variations in individual susceptibility, but anyone who takes an opiate for a long enough time will become addicted" (Berridge \& Edwards, 1987, p. 281).

Secondly, there is, we would argue, the problem of recreational drug use being interpreted as a carefree, thoughtless form of drug use. There exists a tradition of psychedelic drugs being used for the purpose of introspection and self-exploration (Ott, 1993). The introduction of 'recreational' as a descriptor implies that this has been replaced by a consumerist model of drug use, where behaviour is primarily driven by hedonism and less so by intentions towards drug-induced introspection. This is a position we will return to later in this article.

Our own suggestion for a term to cover non-problematic drug use is of course not without its flaws, contradictions and confusions. Unobtrusive drug use could simply refer to chaotic users who have not sought treatment or been identified by health agencies. We would argue in return that such drug use is extremely obtrusive to the individual concerned. Similarly, criminally orientated drugs users who remain out of reach of the drug treatment system are highly obtrusive to those whose houses they break into. Unobtrusive drug use, therefore, needs to be characterized by patterns of use that are neither dominating the lives of those who are taking the drugs in question, nor distressing or damaging to those around them. And we would return to our original position that unobtrusive drug use should, in principle, be applicable to any psychoactive drug. ${ }^{3}$

A second theme in this article could be described as the use of unobtrusive drugs. Put simply, there are a range of psychoactive substances, which only a minority of people - including the wider population of drug users - are likely to have even heard of, and which will have only actually been used by a minority of this group. As an example of this, perhaps the reader would care to trawl through the contents of a whole range of addiction journals and look for references to Fly Agaric. However, whether use of these

\footnotetext{
${ }^{3}$ We are excluding drugs used for psychiatric purposes from this argument. It should also be pointed out that use of psychoactive drugs by people suffering from severe mental health problems is outside the scope of this article.
} 
relatively obscure substances can still be categorized as unobtrusive drug use hinges, as with the drugs discussed above, on the degree of harm and distress which results to the individual and those around them. As such, we now move to the position that unobtrusive use should, in principle, apply to any drug. But to fully test this argument, it is necessary to apply it to a range of drugs that receive scant attention in mainstream drug research. It should also be borne in mind that, as discussed below, certain of these substances have a reputation of being potentially dangerous if used in a thoughtless, reckless manner.

There exist a range of substances naturally occurring in flora, fungi and even animals that have traditionally been used with the purpose of inducing visions and profound shifts of consciousness. While the active compounds themselves vary greatly, the desired effects are very similar: hallucinations, near-death and/or out-of-body experiences and extreme intoxication (Ott, 1993; Schultes \& Hofmann, 1992).

Historically, in tribal communities these were used (e.g.) to communicate with the spirits of the dead or with 'higher beings'. It was understood that to do so involved an element of risk and therefore these rituals were undertaken by adepts trained over a number of years specifically for this purpose (Schultes \& Hofmann, 1992; Weil, 1998).

In the European tradition, common plants such as Deadly Nightshade, Henbane and Mandrake were used. In the popular mind these have associations with witchcraft and black magic. They contain high concentrations of tropane alkaloids and can induce a state of total oblivion accompanied by intense audio-visual hallucinations. Given that the preparation process of both Deadly Nightshade and Henbane is simple (e.g., the leaves of either can be dried and smoked either on their own or mixed with tobacco) it is of interest that their use is not more widespread (Ott, 1993; Schultes \& Hofmann, 1992). Certainly, this is not due to lack of availability, as both Henbane and Deadly Nightshade are not only abundant, but can also be obtained free of charge by the determined experimenter.

Speculatively, this may be due to the rather fearsome reputation these plants have in the popular psyche, particularly as poisons. Alternatively, it has been suggested that this aspect of the use of these plants has been lost to western drug users (Rudgley, 1993) and replaced by a more hedonistic and consumerist pattern of consumption involving laboratory produced analogues (Shulgin, 1997). Possibly because of the synthetic ('designer') nature of these substances, the casual user regards them as safer, more convenient, (and perhaps more 'modern') than their source plants.

Fungi are another widely occurring source of intoxication in Northern Europe. The Liberty Cap mushroom appears every autumn, and can contain high concentrations of psilocybin. The intensity of experience is dependent on the amount consumed together with the set and setting. What is unusual about this substance is that their use has been established in the general UK drug scene for a number of years. The main danger with this hallucinogenic mushroom is poisoning, usually through misidentification or overindulgence.

Associated with these is the Amanita muscaria (Fly Agaric) mushroom. These have been used for visionary purposes for millennia, and are possibly the oldest hallucinogen known to man (Ott, 1993). Over that time it has also acquired a reputation for inducing extreme intoxication and hallucinations. With a reputation of the sort enjoyed by Fly Agaric, one would expect it to be more widely used in contemporary drug and youth culture. However, this does not appear to be the case and even anecdotal evidence for its current use is thin. There are three possibilities for why this might be.

The first is availability. While psilocybin mushrooms can usually be found in hundreds (if not thousands), Fly Agaric is relatively uncommon and therefore harder to find. The second possibility is that, as with the tropane-rich plants, Muscaria is eschewed due 
to the perceived toxicity, intensity and duration of effect. For example, the drinking of the urine of intoxicated individuals has been recorded. In the 21 st century, there is a myth that such recycling resulted from a need to dilute the strength of the mushroom. In actuality, it appears that this practice occurred mainly when mushrooms were scarce (Schultes \& Hofmann, 1992). Thirdly, it has been suggested that there is a folk memory in Europe particularly connected with Fly Agaric, which powerfully associates it with the supernatural and - as with the tropane-rich plants discussed above - witchcraft and black magic (see, e.g., Heinrich, 1995).

Folk tales aside, it is true that these plants are indeed highly toxic, and therefore casual (or poorly researched) use is inadvisable.

In the New World, while plants containing tropane alkaloids (such as Datura and Thorn Apple) were known about and used, the preference appears to have been for plants rich in tryptamines. No doubt this situation arose due to the abundance of such plants (Ott, 1993) in Central and South America.

A mixture of a number of different plant and animal extracts, Yage or Ayahuasca was once indigenous to Amazonia. This is commonly regarded as one of the most intense and long lasting visionary experiences available (Burroughs \& Ginsberg, 1975; Shulgin, 1997), apparently requiring years of instruction in its use by older, experienced users. This, together with more practical factors such as scarcity and cost appear to have precluded widespread use of this drug in the UK.

However, since the 1990s there has been considerable interest in Ayahuasca and a number of publications have appeared detailing its preparation from a number of more readily available plant sources (e.g., Dekorne, 1994; Ott, 1993). In addition to this, a number of websites have also appeared - based in the UK - catering to this specific market (e.g., www.gnosticgarden.co.uk) and offering a wide range of seeds, cuttings and extracts of the plants referred to in these books. As might be expected, the services offered by these websites are variable (as is the monetary cost). Some sell the plants only, whereas others provide the plants together with details regarding dosage and safety. Again, the quality of the information given is variable. Some also provide selected bibliographies, allowing the serious users to conduct their own further research. At the time of writing, every site carried a disclaimer abdicating the supplier of any responsibility for any accidents or health problems arising from the use of these substances.

Other than tryptamines, the Americas are home to indigenous plants rich in phenylethylamines. Peyote and San Pedro cactus contain phenylethylamine compounds one of which, mescaline, is responsible for the hallucinogenic effects. Phenylethylamines have, in a respect, already proliferated far beyond their native borders. MDMA and MDA, for example, are phenylethylamines. These are two out of many (Shulgin, 1991).

Salvia divinorum is another plant originally found only in Mexico, but which is now widely available. The reports on Salvia vary, and some people can have a full-blown visionary experience while others seem to be unaffected (Pendell, 1995). Until the mid-1990s, Salvia would seem to have been largely unknown in the western drug milieu, but as with Ayahuasca there have been a number of dedicated websites, which have appeared over the past few years. Marketed as a 'legal high', Salvia can be purchased as dried or fresh leaf or in the form of a concentrated extract. As well as this, whole plants can also be purchased.

So, can use of the substances described above be classed as 'unobtrusive'? As powerful as these plant-based drugs are, there is as yet very little evidence to indicate their widespread use. Certainly, the drug treatment services aren't overflowing with (e.g.) tryptamine users. Possibly, there is a nascent subculture based around these substances where the users are 
educated as to not only the principles of drug, set and setting, but are also knowledgeable as to the historical and cultural contexts of their use. The proliferation of websites and popular publications promoting and trading in these plants may indicate this to be the case; after all, why start even a small business if there isn't a market?

Perhaps it is useful at this point to return to the Drug, Set and Setting framework. A conclusion to be drawn from the argument presented in this article is that Set is a crucial factor in controlled, recreational, unobtrusive drug use. As stated at the beginning of this article, we would argue that being knowledgeable, thoughtful and well-prepared for using drugs, pretty well any drug, minimizes the risks and dangerousness of the experience, and maximizes positive aspects of the experience.

The role of Setting is also of crucial importance for this position, requiring further exploration and discussion than within the focus of this article. One can identify some examples of drug use, whether widespread or more obscure, within particular settings that are clearly not unobtrusive both in terms of personal risk and social disruption e.g., injecting heroin in a car park, or using Fly Agaric in a crowded club. A further development of this argument would be that use of each and every psychoactive drug has environments conducive to low risk drug use. Whether such an environment is understood and used by individual drug users is crucial, and in many ways brings us to the argument of set as being the typically dominant factor in unobtrusive drug use. We are now a long way from a reductionist model of drug use that is dominated by pharmacology. If the "set" and "setting" are correct, the "drug" itself becomes largely irrelevant and can be used with a higher degree of safety, with the effects being as intended for and expected by the user.

One final point. We are aware that many of the ideas proposed in this article will go against prevailing opinion, particularly the argument that drug use can be, in principle, generally non-problematic to the user and those around them. Being unpopular does not, however, make an argument necessarily flawed. One weakness of this article which the authors would accept is that it is built on a relatively scant empirical literature. The possibilities for research that is genuinely interesting, as well as being a valid and useful addition to the existing drug use and addiction literature are substantial. The authors of this article would be pleased to see more extensive research to replicate existing work, for example on controlled, recreational, unobtrusive heroin use. We would also argue for systematic scientific research to investigate some of the more speculative arguments and conclusions presented above in relation to the use of consciousness-altering plants and fungi. There is a huge space to fill in current drug research.

\section{References}

Alexander, B. (1994). Do heroin and cocaine cause addiction? In P. Brisson (Ed.), L'usage des Drogues et la Toxicamanie (Vol. 2). Montreal: Gaetin Mouin.

Alexander, B. (2000). The globalization of addiction. Addiction Research, 8(6), 501-508.

Berridge, V., \& Edwards, G. (1987). Opium and the people. New Haven: Yale University Press.

Bielman, B., Diaz, A., Merlo, G., \& Kaplan C. D. (1993). Lines across Europe: Nature and extent of cocaine use in Barcelona, Rotterdam and Turin. Amsterdam: Swets and Zeitlinger.

Blackwell, J. S. (1983). Drifting, controlling and overcoming: Opiate users who avoid becoming chronically dependent. Fournal of Drug Issues, 13(2), 219-235.

Burroughs, W. S., \& Ginsberg, A. (1975). The yage letters. San Francisco: City Lights.

Cohen, P., \& Sas, A. (1994). Cocaine use in Amsterdam in non deviant subcultures. Addiction Research, 2(1), 71-94.

Davies, J. B. (1992). The myth of addiction. Chur: Harwood.

Dekorne, J. (1994). Psychedelic shamanism. Portland: Loompanics Unlimited. 
Falk, J. L. (1983). Drug dependence: Myth or motive? Pharmacology, Biochemistry and Behaviour, 19, $385-391$. Goode, E. (1969). Marijuana and the politics of reality. Fournal of Health E Social Behaviour, 10(2), 83-94.

Gossop, M. (1993). Living with drugs. London: Ashgate.

Hammersley, R., \& Ditton, J. (1994). Cocaine careers in a sample of Scottish users. Addiction Research, 2(1), 51-70.

Hammersley, R., \& Reid, M. (2002). Why the pervasive addiction myth is still believed. Addiction Research and Theory, 10(1), 7-30.

Heinrich, C. (1995). Strange fruit: Alchemy, religion and magical foods. London: Bloomsbury.

Mugford, S. K. (1994). Recreational cocaine use in three Australian cities. Addiction Research, 2(1), 95-108.

National Institute on Drug Abuse (2000). Heroin abuse and addiction. Maryland: NIH Publications.

Ott, J. (1993). Pharmacotheon: Entheogenic drugs, their plant sources and history. Kennewick: Natural Products Co.

Pendell, D. (1995). Pharmako/Poeia: Plants powers, poisons, and herbcraft. San Francisco: Mercury House.

Reinarman, C., Murphy, S., \& Waldorf, D. (1994). Pharmacology is not destiny: The contingent character of cocaine abuse and addiction. Addiction Research, 2(1), 2136.

Robins, L., Compton, W., \& Horton, J. (2000). Is heroin the worst drug? Implications for drug policy. Addiction Research, 8(6), 527-548.

Rudgley, R. (1993). The alchemy of culture: Intoxicants in culture. London: British Museum Press.

Shewan, D., Dalgarno, P., Marshall, A., Lowe, E., Campbell, M., Nicholson, S., et al. (1998). Patterns of heroin use among a non-treatment sample in Glasgow (Scotland). Addiction Research, 6(3), 215-233.

Shewan, D., \& Dalgarno, P. (2005). Evidence for controlled herion use? Low levels of negative health and social outcomes among non-treatment users in Glasgow (Scotland) British fournal of Health Psychology, 10(1), 33-48.

Schaler, J. A. (2000). Addiction is a choice. Chicago: Open Court Publishers.

Schultes, R. E., \& Hofmann, A. (1992). Plants of the gods: Their sacred, healing, and hallucinogenic powers. Rochester: Healing Arts Press.

Shulgin, A. (1991). PIHKAL: A chemical love story. Berkeley: Transform Press.

Shulgin, A. (1997). TIHKAL: The continuation. Berkeley: Transform Press.

Waldorf, D., Reinarman, C., \& Murphy, S. (1991). Cocaine changes: The experience of using and quitting. Washington, D.C.: The Drug Abuse Council.

Weil, A. (1998). The marriage of the sun and moon: A quest for unity in consciousness. New York: Houghton Mifflin.

Wise, R. A. (1990). The role of reward pathways in the development of drug dependence. In D. J. K. Balfour (Ed.) Psychotropic drugs of abuse. New York: Pergamon.

Zinberg, N. (1984). Drug, set and setting: The basis for controlled intoxicant use. New Haven: Yale University Press. 
Copyright of Addiction Research \& Theory is the property of Brunner / Routledge and its content may not be copied or emailed to multiple sites or posted to a listserv without the copyright holder's express written permission. However, users may print, download, or email articles for individual use. 